\title{
TTR
}

Traduction, terminologie, re?daction

\section{Le travail sur la lettre : politique de décentrement ou tactique de réappropriation?}

\section{Gillian Lane-Mercier}

Volume 11, numéro 1, 1er semestre 1998

Diachronie et synchronie

Diachronics and Synchronics

URI : https://id.erudit.org/iderudit/037316ar

DOI : https://doi.org/10.7202/037316ar

Aller au sommaire du numéro

\section{Éditeur(s)}

Association canadienne de traductologie

ISSN

0835-8443 (imprimé)

1708-2188 (numérique)

Découvrir la revue

Citer cet article

Lane-Mercier, G. (1998). Le travail sur la lettre : politique de décentrement ou tactique de réappropriation? TTR, 11(1), 65-88. https://doi.org/10.7202/037316ar

\section{Résumé de l'article}

Le travail sur la lettre : politique de décentrement ou tactique de réappropriation? - La visée de cet article est double. D'une part, il s'agit de répondre à quelques-unes des attaques récentes dirigées par Douglas Robinson contre les pratiques traductionnelles littérales, que Robinson qualifie de « protofascistes ». D'autre part, il s'agit d'interroger, dans le cadre d'un projet de retraduction littérale de The Hamlet de William Faulkner mené à l'université McGill entre 1990 et 1996, la valeur épistémologique, éthique et méthodologique de l'approche littérale prônée par Antoine Berman, en vue à la fois d'une remise en question et d'une reconceptualisation de cette dernière, dont l'élitisme et l'idéalisme ont déjà été soulignés. Pour ce faire, nous proposons une analyse des présupposés politico-idéologiques véhiculés, d'un côté, par le recours au vernaculaire rural du Québec pour traduire le parler non-standard des personnages faulkneriens, et de l'autre, par les stratégies de survernacularisation et de dévernacularisation successivement mobilisées à ces fins. Si, à première vue, ces stratégies correspondent, respectivement, à une politique de décentrement (littéralisme, non-annexion de l'Autre socio-culturel) et à une tactique de réappropriation (hypertextualité, domestication de l'Autre socio-culturel), l'analyse démontre jusqu'à quel point une telle distinction est peu opératoire sur les plans épistémologique, éthique et heuristique. En effet, le choix de recourir au vernaculaire rural québécois pour respecter l'étrangeté du texte source engage simultanément, selon un rapport d'implication mutuelle, le littéralisme et la domestication, dont la finalité commune consiste à refuser l'ethnocentrisme radical en déstabilisant les attentes du lecteur.
Tous droits réservés (C TTR: traduction, terminologie, rédaction — Les auteurs, 1998
Ce document est protégé par la loi sur le droit d'auteur. L'utilisation des services d'Érudit (y compris la reproduction) est assujettie à sa politique d'utilisation que vous pouvez consulter en ligne.

https://apropos.erudit.org/fr/usagers/politique-dutilisation/ 


\section{Le travail sur la lettre : politique de décentrement ou tactique de réappropriation?}

\section{Gillian Lane-Mercier}

Dans son nouveau livre intitule What is Translation?, Douglas Robinson qualifie toute pratique traductionnelle littérale de * protofasciste " (Robinson, 1997, p. 90). Fondées, on le sait, sur une politique de décentrement qui vise à respecter l'altérité et le prestige du texte source grâce à la déstabilisation de l'horizon d'attente des lecteurs et des multiples institutions culturelles sur lesquelles cet horizon d'attente s'embraye, les traductions littérales ne sont rien d'autre, selon Robinson, qu'une " celebration of unabashed cultural elitism, scorning the 'masses' and their demand for instant understanding * (p. 82), " an implicitly political move, an attempt [...] to gain political ascendancy for our group over [those] who have peripheralized us " (p. 90). Et tout en admettant que les notions de l' $\alpha$ étranger " et de l'a étrangeté " proposées par le très influent tenant du nélittéralisme contemporain, Antoine Berman, amènent ce dernier à récuser un littéralisme trop opaque, trop illisible (trop fasciste?) au profit d'une conception éthique de l'acte de traduire comme geste anti-annexionniste susceptible d'instaurer un dialogue entre 
soi (le propre) et l'autre (l'étranger) ${ }^{1}$, Robinson fustige ce qu'il appelle * the coercive politics " (p. 87) de la traductologie, telle que Berman l'a définie dans $L$ 'épreuve de l'étranger. Déclaration de Berman :

[...] l'un des axes de [la traductologie] est d'élaborer une théorie de la traduction non ethnocentrique dont le champ d'application est generalisé. Cette thérie est simultanement descriptive et normative.

Elle est descriptive, dans la mesure où elle analyse très précisément les systèmes de déformation pesant sur toute opération de traduction et peut, à partir de cette analyse, proposer un contre-système. Normative, dans la mesure où les alternatives qu'elle définit au sujet du sens de la traduction sont contraignantes. (Berman, 1984 , p. 297)

Riposte de Robinson :

Berman wants to control translation as absolutely as the normative system that has reigned in the West since Augustine and Jerome [...] by instilling in translators' heads a generalized operating system that will guide them to empirically correct decisions without having to "descend" [...] to the infinitude of contextual variations. (p. 88)

Qui pis est, l'étrangeté dont se lestent les traductions néolittérales en général et celles prônées par Berman en particulier signale moins la

1 On notera cependant que le caractère conciliateur de cette remarque est immédiatement miné :

[...] Berman is adamant about not prescribing literalism; his foreignism looks a bit like literalism, reads like it, but differs from it in his willingness to compromise with assimilationist ideals, to write a readable and vigorous target language that nevertheless retains some trace of the source-language text's strangeness, otherness, foreignness, alterity. Some might call this timid literalism, literalism without the courage of its convictions [...]. (p. 84)

Robinson poursuit sa critique en s'etonnant de ce refus, de la part de Berman, d'un litteralisme radical et en y voyant une éventuelle contradiction par rapport à sa théorie, sachant que par ailleurs Berman refuse explicitement de tenir compte des compétences des lecteurs : $\alpha$ If he is as unconcerned about reader response as he claims to be, what possible objection can he have to 'opaque' literalism? * (p. 85) 
présence de l'Autre socio-culturel que celle de rapports de pouvoir immanents aux discours dominants de la société cible. C'est dire que si les incongruités linguistiques et stylistiques du texte traduit sont a priori tributaires d'une stratégie plus ou moins radicale de décentrement et de métissage, elles relèvent en fait, d'après Robinson, d'une subtile rhétorique de la punition, d'un * interlangage * artificiel, condescendant et aliénant créé de toutes pièces par le traducteur " to bridge the gap between the writer's world and the reader's world in subtly irritating ways " (p. 94). Et Robinson de conclure :

Foreignism, it turns out, isn't foreign at all [...]. It's the language of authorities imposing an alien set of behavioral norms on a subordinate group - a condescending doubletalk that wields alterity like a velveteen stick [...,] an authoritarian discourse drawing on rhematic and rhetorical repertoires of foreign languages and alien registers of the native language in order to mystify priestly power. (p. 94)

Robinson ne conteste nullement le versant proprement éthique du littéralisme; bien au contraire, * the translator's job should be to wake people up, to rub their noses in the unknown, the uncanny, the strange and the foreign * (pp. 93-4). Ce qu'il conteste, ce sont les présupposés politico-idéologiques que, selon lui, tout projet littéral véhicule. D'où sa question : $[$ [...] can we agree that neoliteralism, foreignism, the strange feel of the foreign language in the target-language text, is the only, or even the best, way to accomplish that [ethical] goal? n La réponse : \& I think not. In fact, I think it is one of the worst " (p. 94). Infiniment plus efficace dans sa visée iconoclaste et, du coup, éthique, l'appropriation radicale du texte source est le seul moyen, selon Robinson, de sortir le lecteur de sa " torpeur " endémique et, ce faisant, de réaliser la finalité éthique que le littéralisme bermanien se donne sans l'atteindre :

[...] readers are shaken far more effectively out of their "stupor" - if we have to follow the neoliteralists in positing a stupefied reader - through radical and aggressive domestication of the source-language text than through timid foreignism, which is one of the most powerful stupefactants around. (pp. 94-5)

De telles attaques donnent à réfléchir, à plus forte raison lorsqu'on a participé, comme c'est mon cas, à un groupe de recherche dont le projet consistait, précisément, à proposer une retraduction littérale du Hamlet de 
Faulkner ${ }^{2}$. Sauf exception, il n'est jamais agréable de se voir soupçonné de protofascisme. Aussi aimerais-je revenir sur certains tenants et aboutissants de la pratique traductionnelle préconisée par le groupe, en vue 1) de rendre compte de ce que je crois être l'un des apports les plus originaux - et les plus inattendus - du projet, soit la remise en cause et la reconceptualisation de la notion bermanienne de traduction littérale, et 2) de répondre, ne serait-ce qu'indirectement, aux critiques de Robinson en examinant les raisons de cette remise en cause et la nature de la reconceptualisation qui en a découlé.

Deux mots, pour commencer, à propos du projet traductif du groupe. Ce projet se voulait la combinaison de deux approches à priori complémentaires. D'une part, il s'agissait de procéder, à l'instar de Tophoven, à une retraduction dite transparente du texte de Faulkner, c'est-à-dire à l'élaboration d'un appareil paratextuel relativement étoffé qui, coextensif à la retraduction proprement dite, avait pour fonction première d'expliciter en les commentant les choix et les stratégies traductionnels. D'autre part, il s'agissait de réaliser une retraduction littérale, inspirée de Berman, dont l'objectif principal était de restituer, grâce au recours au vernaculaire rural québécois, le parler non-standard des " country whites ", lequel avait été presque entièrement gommé dans la seule traduction francaise du Hamlet, celle de René Hilleret publiée en 1959. La complémentarité des approches résidait, entre autres, dans la possibilité d'inclure au niveau du paratexte des éclaircissements quant au sens des termes et expressions vernaculaires - tant américains que québécois, d'ailleurs - et dans la manière dont les deux approches perturbaient, chacune à sa façon, les pratiques traductionnelles dominantes fondées sur l'hypertextualité et l'invisibilité du sujet traduisant.

Tel était le projet traductif initial du groupe. Or, ces choix ont tôt fait de soulever des enjeux considérables, dont certains seront évoqués tantôt, de sorte que le groupe a été peu à peu amené à repenser ses stratégies traductionnelles. Dans un premier temps, le paratexte a été

2 Il s'agit du Groupe de recherche en traductologie (GRETI), fondé en 1990 au département de langue et littérature françaises de l'Université McGill, subventionné par le Conseil de recherches en sciences humaines du Canada. 
éliminé, ce qui a eu pour effet immédiat de provoquer, outre une diminution de la lisibilité des réseaux vernaculaires, l'abandon de l'approche transparente. Aussi je n'y reviendrai pas. Dans un deuxième temps, une politique de dévernacularisation a été adoptée afin de corriger le caractère survernacularisé des tentatives de retraduction initiales. Cette politique a eu pour résultat direct l'accroissement de la lisibilité des réseaux vernaculaires et, pour résultat indirect, la remise en question de l'approche littérale ${ }^{3}$. C'est à cette dernière que je voudrais maintenant m'attarder, afin de faire ressortir les fondements politico-idéologiques et éthiques des choix et stratégies successivement mobilisés par le groupe pour traduire le parler non-standard des personnages faulknériens.

Je partirai de deux constatations. Première constatation : selon les membres du groupe, l'étrangeté du texte faulknérien était tributaire, pour une large part, de l'emploi du vernaculaire. Une traduction littérale en impliquait donc le maintien. Deuxième constatation, nettement plus problématique : non seulement les différentes solutions retenues à tour de rôle pour traduire le parler non-standard du Hamlet paraissaient, pendant un temps, légitimes, mais toutes, en dépit de leurs dissemblances parfois flagrantes, visaient la traduction littérale du vernaculaire de départ et s'inscrivaient, par là, dans une politique, non annexionniste et non ethnocentrique, de décentrement. D'où l'importance de s'interroger sur la valeur épistémologique, éthique et méthodologique de l'approche bermanienne; interrogation qui me semblait d'autant plus urgente que la politique de dévernacularisation envisagée par le groupe risquait de recentrer ce qu'il importait, précisément, de décentrer. Pour ce faire, il est nécessaire d'évoquer quelques-uns des enjeux soulevés par le choix du vernaculaire rural québécois, ce qui m'oblige à une digression.

Rappelons dans un premier temps que l'identité des Québécois repose essentiellement sur des critères linguistiques ${ }^{4}$. C'est grâce à la fois

${ }^{3}$ Il faut toutefois préciser que, si le groupe a cédé aux contraintes de lisibilité pour les dialogues en vernaculaire, il n'a pas reculé devant * l'etranger * dans la narration (voir Chapdelaine et Lane-Mercier, à paraitre).

${ }^{4}$ Je suis parfaitement consciente du caractère extrêmement général et, par là, réducteur des remarques qui composent la présente parenthèse. Elles visent uniquement à esquisser l'arrière-fond axio-idéologique du développement qu'elles 
à la langue qu'ils parlent et à la manière dont ils la parlent qu'ils se définissent comme communauté culturelle distincte des communautés anglophones d'Amérique du Nord et de celles de la francophonie. Or, on sait sans doute que dès la fin du XIX' siècle leur manière de parler le français fut stigmatisée par les Canadiens anglais et les Américains, qui le caractérisèrent de " patois, incompréhensible pour les Français et, a fortiori, pour les étrangers ayant une connaissance du français. A ce French Canadian Patois, on opposa dès lors le Parisian French [...] . (Bouchard, 1990, p. 36). On sait également qu'à la querelle du French Canadian Patois qui s'ensuivit entre anglophones et Canadiens français, succéda à partir de 1960 la querelle du joual, parler populaire des classes ouvrières urbaines fortement contaminé par l'anglais que les Canadiens français eux-mêmes opposaient " non plus au français de Paris, mais à une nouvelle variété moins étroitement identifiée à des schémas culturels précis : au français international, notion des plus abstraites " (Bouchard, 1990, p. 45).

Mais on connaît peut-être moins les réactions des Canadiens français face à ces phénomènes de stigmatisation de provenance externe et interne, que Chantal Bouchard $(1988,1990,1998)$ a analysées à partir d'articles de journaux parus dans la presse québécoise entre 1878 et 1970. Selon elle, ces réactions ont oscillé entre un sentiment d' "inșécurité linguistique [...] apparemment plus aigu chez les lettrés d'ailleurs que dans les autres couches de la population $n$ et $~$ l'apparition et la disparition [d']attitudes de défense systématique de la langue des Canadiens français [...] " (1990, p. 37). Ainsi, en schématisant à l'extrême, entre 1880 et 1910 les Canadiens français entretinrent une opinion plutôt négative de leur langue; opinion qui fut remplacée entre 1910 et 1945 , alors que la querelle du French Canadian Patois battait son plein, par une attitude plus positive qui visait à parer aux attaques des anglophones en leur démontrant que la langue franco-canadienne était bien le français. Ce n'est qu'après 1940, au moment où la querelle du French Canadian Patois s'essouflait et que débutaient les grands mouvements d'urbanisation et d'industrialisation, que de nombreux Canadiens français, inquiets de l'état de détérioration de leur parler au contact de l'anglais, prirent le parti de préconiser l'emploi d'un français plus standard, plus $\propto$ international $»$. Or, la présence

servent à introduire. Pour un excellent aperçu de la question, voir Bouchard 1998. 
de plus en plus visible du joual dans les appareils culturels du Québec (médias, écoles, littérature) ${ }^{5}$ au cours des années 60, si elle fut décriée par ses détracteurs, coincida avec une attitude à la fois positive et militante de la part de ses défenseurs, qui situèrent le joual au centre de leurs luttes socio-politiques et culturelles en revendiquant pour lui le statut de langue nationale québécoise.

Cela dit, il faut tenir compte des valeurs proprement culturelles dont témoignent ces attitudes tantôt négatives, tantôt positives, tantôt mitigées, ambiguess ${ }^{6}$; attitudes qui sont tributaires de la dichotomie, hautement idéologique, entre langue dominante (l'anglais, le français de France) et langue dominée (la langue du Québec) :

[...] un individu qui juge mal sa façon de parler [...] appartient aux classes dominés de sa communauté et il partage les jugements de valeurs qui définissent les normes de prestige d'après le sociolecte de la classe dominante. Son propre sociolecte est stigmatisé socialement et il admet ce jugement. (Bouchard, 1988, pp. 7-8)

D'une part, les valeurs culturelles véhiculées par de telles attitudes sont d'ordre socio-économique et politique. C'est ainsi par exemple que, voyant leurs revendications politiques et civiques remises en question tant qu'ils n'ont pas amélioré leur * français pouilleux ( ( lousy French $*-$ Pierre Elliot Trudeau, ministre fédéral de la justice ${ }^{7}$ ), les Québecois répondent en accélérant l'implantation de nouvelles législations linguistiques :

${ }^{5}$ Cette présence était double : d'une part, il s'agissait d'un discours sur le joual; de l'autre, de l'emploi du joual, par exemple dans les écrits littéraires des écrivains du groupe Parti Pris et dans des séries télévisées.

${ }^{6}$ En anticipant, il est important de souligner que, tout en défendant leur langue, les lettrés ne cherchaient ni à l'enseigner, ni à la faire entrer, autrement que par petites touches stylisées, dans la littérature. La langue de référence, dans ces domaines, reste le français standard de France. Voir Bouchard, 1998, pp. 141, 144-46, 222-24.

1 Anon., « Trudeau : A Québec d'améliorer d'abord son 'mauvais français' ", Le Devoir, 15-2-68, cité dans Bouchard, 1990, p. 47. 
C'était bien, en effet, ce genre de raisonnement que les Canadiens français craignaient depuis un demi-siècle : qu'on prenne prétexte du soi-disant patois pour réduire leurs droits politiques et les pousser irréversiblement dans la voie de l'assimilation. Puisqu'on les attaquait sur le terrain linguistique, les Québécois, tout compte fait, prirent les mesures politiques qui s'imposaient pour protéger leur langue d'une plus grande contamination par l'anglais. (Bouchard, 1990, p. 48)

D'autre part, ces valeurs culturelles sont partie intégrante de ce que Sherry Simon appelle $*$ a history of the linguistic expressions of alterity $*(1992$, p. 168). Le parler franco-canadien, comme le joual, ont contribué à la construction d'identités - de soi, de l'autre - définissables en termes de formations discursives pourvues $d^{\prime}$ ' un ensemble de règles anonymes, historiques "(Foucault cité dans Brisset, 1990, p. 23) qui, se manifestant sous forme de topoi, de représentations, de schèmes argumentatifs, de scénarios préformés, déterminent ce qui peut et doit être dit à un moment historique donné.

C'est ainsi que de multiples discours circulant depuis le $\mathrm{XIX}^{e}$ siècle sur la langue et le peuple franco-canadiens ont peu à peu convergé pour construire, vers 1910, le double topos du paysan Canadien français comme * un être fruste, bon et simple, pour ne pas dire simplet, une sorte de 'bon sauvage' "(Bouchard, 1988, p. 11) et de son parler comme l'incarnation des vraies valeurs sociales et spirituelles. * Le paysan devient, idéologiquement, le dépositaire de toutes les vertus de la race " (Bouchard, 1998, p. 119). Adoptée aussi bien au Canada anglais qu'au Canada français, cette image du Canadien français servait toutefois des intérêts antagonistes ${ }^{\mathbf{B}}$ : si les écrits et traductions du Canadien anglais William Hume Blake, par exemple, relevaient d'un " elegant propagandizing in favor of the nobility and archaic virtues of French

'En effet si les élites canadiennes françaises s'acharnent tant à combattre le mythe du French Canadian Patois propagé par les Canadiens Anglais et les Américains, c'est bien parce qu'il :

[...] sous-entend l'ignorance, l'inculture, une forme de déchéance, il tend à isoler les Canadiens français de leurs origines, à leur nier le prestige qui s'y attache; cela permet de remettre en doute les institutions, en particulier l'instruction, et l'utilité de perpétuer les droits politiques du français au Canada. (Bouchard, 1998, p. 147) 
Canada * qui plongeait le lecteur * into a genteel and patemalistic universe " (Simon, 1992, p. 163), en revanche, le désir de défendre leur * patois incompréhensible * des attaques qui, entre 1910 et 1940, n'ont cessé de fuser, a amené les Canadiens français à forger des tactiques argumentatives visant à asseoir sa légitimité :

On assiste donc, à partir de 1910, à une vaste réhabilitation de la classe paysanne, et de sa langue surtout menée par des lettrés dont les objectifs consistent, semble-t-il, à combattre la détérioration de l'image que les Canadiens français se font d'eux-mêmes. [...] ii est désormais urgent d'abandonner l'autocritique et de consolider, voire de reconstruire une image positive. (Bouchard, 1998, pp. 120-21)

A ces fins, on appuie la thèse selon laquelle la langue franco-canadienne est celle de Louis XIV, accordant une axiologisation positive à ses particularités linguistiques dont il faut conserver $\alpha$ les mots les plus savoureux, les expressions les plus imagées " (Bouchard, 1988, p. 12). On multiplie les recherches étymologiques, on glorifie l'authenticité du parler paysan, on procède à des amalgames de lieux communs issus de discours connexes - ceux de l'Église (" apôtre de la langue *), de la famille ("vieux français de nos pères *), de la noblesse (* sa majesté la langue française $n^{9}$ ) - dont la fonction rhétorique consiste à maintenir les valeurs traditionnelles et passéistes. On a affaire à :

[...] une espèce de fétichisation de la culture traditionnelle, à ia construction d'une identité réactionnelte dont l'élaboration vise d'abord à permettre aux Canadiens français de résister à l'érosion [sociale]. On s'appuie sur ce qui semble le plus solidement acquis, ce qui n'est pas remis en cause par les Anglo-Canadiens, sur la valeur commune et distinctive des Canadiens français, leur catholicisme. (Bouchard, 1998, p. 129)

Enfin, on dénonce la lourde présence d'anglicismes qui caractérise aussi bien le parler populaire des villes industrialisées que la langue des classes

${ }^{9}$ Exemples cités dans Bouchard, 1988, p. 16. 
bourgeoises $^{10}$, tous deux se trouvant dès lors opposés non seulement au français international, mais aussi au français des paysans, perçu comme plus pur car non * perverti " par l'anglais.

Cependant, avec l'essor de l'urbanisation, « on oublie rapidement les paysans, classe réduite en nombre et surtout en importance dans l'identité québécoise * (Bouchard, 1988, p. 17). Non seulement on se rend compte que \& la culture de l'ouvrier urbain n'a plus grand-chose de commun avec celle du traditionnel habitant dont on avait tant vanté les vertus pendant trente ans n (Bouchard, 1998, p. 133), mais, plus grave encore, l'anglicisation finit par toucher la classe paysanne elle-même :

Le discours d'exaltation du français des paysans en vient [...], malgré les intentions initiales qui étaient de légitimer la langue des Canadiens français, à se retourner contre la majorité d'entre eux et à devenir un discours de stigmatisation assez violent. [...] Le moment n'est pas loin où le discours apologétique devient impossible, parce que cela reviendrait à condamner toute la société. (Bouchard, 1998, pp. 138-44)

Ce moment, c'est l'après-guerre. Se manifeste alors, chez les lettrés, une nette préférence pour le français standard, de sorte que même l'emploi littéraire de régionalismes, si applaudi depuis 1910, est récusé :

Je ne me suis jamais si bien rendu compte que ce régionalisme, qui m'avait tant déplu d'abord, est, en définitive, à l'insu de ces auteurs, sans doute, un moyen d'anglicisation. Un moyen indirect d'isoler les Canadiens français, de les condamner à vivre parmi les gens normaux comme un troupeau de sourds-muets. (P. Baillargeon, chronique " Mélanges; l'erreur des régionalistes ", La Patrie, 16 novembre 1947, cité dans Bouchard, 1998, p. 213)

Désormais redevables à une "philosophy of cultural anticolonialism" (Simon) de plus en plus articulée, les luttes linguistiques se déplacent, débordent les seules considérations défensives d'(in)correction et d'(im)pureté linguistiques pour assumer des allures nettement plus

10. La classe instruite [...] sera maintes fois critiquée à partir des années 1935-1940; on lui reproche le snobisme de l'anglicisation et la paresse de ne pas utiliser ce qu'elle a eu la chance d'apprendre contrairement aux autres : le 'bon' français. » (Bouchard, 1988, p. 14) 
offensives. Déclenchée à la faveur d'un climat politique effervescent en 1959 , la querelle du joual s'accompagne, du côté de ses défenseurs toujours plus nombreux, de revendications multiples. Contestation de l'hégémonie du français écrit parisien, création d'un répertoire culturel québécois, légitimation du joual comme langue nationale, reflet de * the aggressive clash of alterities "(Simon, 1992, p. 172) constitutif de la réalité culturelle de l'époque, rejet de la domination politico-économique imposée depuis deux siècles par les Canadiens anglais, affirmation de la spécificité identitaire et culturelle des Québécois" ${ }^{11}$, ces revendications ont pour dénominateur commun la destruction du statu quo, la création d'une société radicalement nouvelle. Nous sommes loin ici des valeurs traditionnelles conservatrices sous-jacentes aux débats linguistiques de la première moitié du siècle ${ }^{12}$.

Pourquoi cette digression? Parce que d'aucuns pourraient voir dans la réutilisation du parler rural québécois du début du siècle pour traduire les réseaux vernaculaires du Hamlet un geste qui ou bien le stigmatise de nouveau en réactivant le mythe aujourd'hui révolu du French Canadian Patois comme parler dégradé, ou bien parodie les valeurs positives dont se lestait le langage du paysan franco-canadien. Cette interprétation serait d'autant plus justifiée que non seulement les " country whites " faulknériens sont dotés de traits caractérologiques essentiellement négatifs - manque d'instruction, ruse, opportunisme, exploitation, dégénérescence, amoralité -, mais c'est entre autres par le biais de la représentation de leur parler non normatif que Faulkner a cherché d̀ créer un certain relief comique. D'autres au contraire pourraient voir dans ce choix un geste réactionnaire qui, en évitant l'emploi đu joual pour renouer avec une vision du monde propre à l'époque de la colonialisation, court-circuite les acquis de la Révolution tranquille et du mouvement anticolonialiste qui l'a portée. Car si le choix du vernaculaire rural québécois est motivé sur le plan proprement littéraire, pour autant qu'il établit des congruences traductionnelles heureuses (le parler des " country whites " du Hamlet remonte à la même époque, est d'ordre rural,

11 Pour un excellent aperçu des pratiques traductionnelles des dramaturges québécois entre 1968 et 1988, voir Brisset 1990.

12 Pour une analyse de la querelle du joual, voir Bouchard, 1998, pp. 229-284. 
paysan, etc.), en revanche sa nature a priori anachronique par rapport à la situation d'énonciation du groupe et de ses lecteurs empiriques déclenche sur les plans historique et idéologique des incongruences plus ou moins massives.

Quoiqu'il en soit, dans un cas comme dans l'autre le projet traductionnel affiché du groupe, et surtout sa dimension explicitement contestataire vis-à-vis des pratiques traductionnelles hypertextuelles, se verraient doublement compromis : loin de résister à l'hégémonie linguistique dont jouit le français standard au sein de l'institution littéraire, la retraduction du Hamlet ne ferait qu'entériner le statut * inférieur " du vernaculaire rural du Québec. Et (ce qui revient en quelque sorte au même) loin de revêtir une efficacité argumentative susceptible de contester les pratiques traductionnelles dominantes afin de donner à lire le texte faulknérien dans toute son étrangeté, l'emploi littéraire du vernaculaire rural, contrairement à l'emploi littéraire du joual qui, lui, comporte les traces d'une résistance culturelle réelle, ne ferait que perpétuer le statu quo socio-idéologique et esthétique que les pratiques traductionnelles dominantes s'efforcent de naturaliser. Historiquement liés à un mouvement non de résistance mais de défense ${ }^{13}$, les discours sur le vernaculaire rural, de même que les imitations littéraires que l'on en proposait, servirent en effet à promouvoir des valeurs (pureté, vertu, patrimoine, noblesse) constitutives des intérêts de nos institutions les plus conservatrices et, à travers elles, du discours dominant.

D'où la question de savoir si le choix du vernaculaire rural québécois ne constitue pas une contradiction idéologique interne du projet de retraduction du groupe, déposant des valeurs réactionnaires au cour même d'un projet qui se veut progressiste. Cette question paraît d'autant plus pressante que, d'une part, on est en droit de se demander jusqu'à quel point les stratégies littérales visant à rendre l'étrangeté du texte source sont en soi plus fidèles à la spécificité culturelle et linguistique de ce dernier. Ne réinscrivent-elles pas tout simplement, au sein du texte source,

13 * Ce militantisme [...] est essentiellement défensif. Les mesures visent à repousser les menaces immédiates [...]. Elles ne s'attaquent pas aux sources mêmes du mal qui sont l'affaiblissement socio-politique et économique de la nation canadienne-française, sa prolétarisation et le manque de prestige qui s'ensuit. * (Bouchard, 1998, p. 127) 
des valeurs marginalisées de la culture cible, servant ainsi des intérêts tout aussi ethnocentriques que les stratégies hypertextuelles? Comme le remarque Françoise Massardier-Kenney, " adapting the radical gesture of the text could very well be another way of making the text 'culturally fluent', of making it fit our own contemporary expectations of what constitutes 'resistant' writing " (1994, p. 15). D'autre part, la politique de dévernacularisation que le groupe a fini par adopter impliquait un processus de normalisation doublé à la fois d'une revalorisation explicite des contraintes de lisibilité et des règles du a bien écrire " (dont le conservatisme est bien connu) et d'une revalorisation implicite des principes mêmes contre lesquels Berman n'a cessé de s'insurger : acceptabilité, accessibilité, conformité aux attentes linguistiques et culturelles des lecteurs cibles, hypertextualité et ethnocentrisme. Il en découlerait une atténuation progressive de la portée contestataire du projet sur le triple plan esthétique, traductologique et pragmatique, pour autant que la lisibilité désormais recherchée par le groupe témoignerait d'un mouvement d'esquive par rapport au projet de départ (déstabiliser les attentes des lecteurs), d'une diminution de l'étrangeté du texte d'arrivée au profit d'un travail de domestication nettement plus évident, et d'un désir de se conformer davantage à l'horizon d'attente des lecteurs tant québécois que non québécois.

Un premier élément de réponse à cette question aux ramifications si multiples est sans doute à chercher dans la situation d'énonciation du groupe. Le choix du vernaculaire rural québécois de la première moitié du $\mathrm{XX}^{\mathrm{e}}$ siècle pour traduire l'anglais non-standard du Hamlet place nécessairement (et minimalement) l'entreprise du groupe a la fois par rapport à l'histoire de la langue française au Québec, par rapport à l'évolution du système axio-idéologique qui informe cette histoire, par rapport aux multiples enjeux - politiques, culturels, institutionnels, économiques, ethniques - qui la constituent, et, enfin, par rapport a l'histoire des pratiques discursives en général et des pratiques traductionnelles en particulier. Les représentations du parler rural québécois du début du siècle ne sauraient en conséquence ne pas s'infuser de valeurs et d'intérêts actuels, pas plus qu'elles ne sauraient constituer des anachronismes de fait. Au lieu de transmettre, dans toute sa pureté et plénitude, un état de langue passé, ces représentations sont * construction de l'intelligible de notre temps "(Barthes, 1964, p. 257), témoignant ainsi d'une position traductive qui conçoit l'acte traductionnel non pas comme 
la reconstitution d'une * origine ", quelle qu'elle soit (texte, auteur, sens, lettre, parler), mais comme un processus historiquement et culturellement déterminé qui s'ancre dans une dialectique de la reproduction et de la transformation. Non seulement le groupe assume pleinement, dans un double mouvement socio-idéologique et éthique, la responsabilité des incongruences historiques et idéologiques que la représentation littéraire d'un parler de l'époque de la colonisation socio-économique du Québec suscite, de même que les éventuelles lectures * à rebours * évoquées plus haut, mais il lui accorde, par le biais d'un projet traductionnel qui se veut anti-institutionnel, progressiste, des valeurs anti-colonialistes de résistance et de contestation habituellement dévolues à la représentation littéraire du joual ${ }^{14}$. Autrement dit, ces incongruences historiques et idéologiques, dès lors transformées en stratégies traductionnelles, sont constitutives du projet de retraduction littérale du groupe.

Deuxième élément de réponse : la politique de dévernacularisation a eu pour résultat capital d'amener le groupe a reconnaître l'idéalisme et l'essentialisme de l'approche littérale de Berman, du moins telle qu'il l'avait définie avant la publication de son dernier ouvrage (1995). Désir d'accéder à * la pure visée * (1984, p. 17), à " l'essence voilée de toute traduction " $(1985 \mathrm{a}$, p. 67) qui consiste à " accueillir l'Étranger comme étranger * (1985a, p. 68), à " accentuer son étrangeté * (1985a, p. 67); refus de tenir compte des attentes du lecteur, la conception bermanienne de traduction littérale conduit néanmoins à la constatation de l'intraduisibilité des sociolectes source. Précisant que :

[t]raditionnellement, il existe une manière de conserver les vernaculaires en les exotisant. L'exotisation peut prendre deux formes. D'abord, par un procédé typographique (les italiques), on isole ce qui, dans l'original, ne l'est pas. Ensuite, et plus insidieusement, on en " rajoute * pour a faire plus vrai ", en soulignant le vernaculaire à partir

\footnotetext{
${ }^{14} D$ 'où les suppléments de sens qui caractérisent les sociolectes traduits : " Notre recréation de la composante dialogale revêt dès lors un caractère innovateur dont était dépourvu le projet sociolectal faulknérien, qui pour sa part s'inscrivait tout à fait dans la tradition littéraire du Sud américain " (Chapdelaine et Lane-Mercier, a paraître). En effet, cette valeur contestataire conférée aux sociolectes du texte d'arrivée est absente des sociolectes du texte de départ, dont les enjeux étaient autres.
} 
d'une certaine image (d'Epinal) de celuj-ci. [...]

L'exotisation peut rejoindre la vulgarisation en s'efforçant de rendre un vernaculaire étranger par un vemaculaire local [...]. (1985a, p. 78),

Berman ajoute aussitôt :

Malheureusement, le vernaculaire, collant au terroir, résiste à toute traduction directe dans un autre vernaculaire. Seules les langues * cultivées n peuvent s'entre-traduire. Une telle exotisation, qui rend l'étranger du dehors par celui du dedans, n'aboutit qu'à ridiculiser l'original. (1985a, p. 78)

Foncièrement élitiste, contradictoire par rapport à ses thèses principales (voir Lane-Mercier 1997), cette remarque à propos de l'" exotisation ridiculisante " dévoile les assises métaphysiques de sa conception de la traduction littérale, ainsi que l'absurdité d'envisager, comme Berman le suggère ici, la possibilité (même impossible!) de rendre l'étranger du dehors par celui du dehors, ou encore un vernaculaire étranger ${ }^{15}$ par un vernaculaire étranger. En choisissant de traduire le parler non-standard des " country whites * par le vernaculaire nural québécois - mais le choix de tout autre vernaculaire aurait abouti au même résultat -, le groupe n'a fait que corroborer le fait que " foreignism [...] isn't foreign at all * (Robinson, 1997, p. 94), que la traduction:

[...] assumes the impossibility of any autonomous cultural value and views the foreign as at once irredeernably mediated and strategically useful, a culturally variable category that needs to be constructed to guide the translator's intervention into the current target-language scene. (Venuti, 1995, p. 205)

Il s'ensuit que le projet du groupe, dans ses formulations initiales comme dans ses formulations ultérieures, récuse cet aspect élitiste du concept de traduction littérale et la contradiction qui la sous-tend. Plutôt

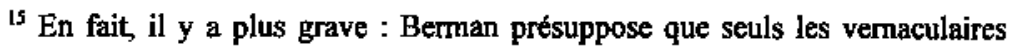
* collent au terroir * et que, en revanche, les a langues cultivées " (ou standard, ou nationales, dont le français, l'anglais, etc.) sont a-contextuelles. Une telle conception est redevable aux théories du langage positivistes, dont l'idéalisme n'est plus à démontrer. 
que de prôner l'intraduisibilité des sociolectes source, le groupe a tout simplement déplacé les impératifs bermaniens d'étrangeté et d'altérité qui, au lieu de se référer aux seuls texte et culture de départ, se rattachent aussi au texte et à la culture d'arrivée. Le choix d'un vemaculaire par nécessité "local ", " du dedans " (car comment en serait-il autrement?) doit son étrangeté et son altérité non pas au fait qu'il permet un accès direct à l'étrangeté et à l'altérité du Hamlet, mais à son statut marginalisé relativement aux institutions culturelles dominantes de la société d'accueil. Le métissage de soi et de l'autre dès lors opéré engage, selon un geste inéluctablement ethnocentrique, les langages standard et nonstandard, dominants et dominés de la culture d'arrivée, de sorte que l'idéalisme intrinsèque à la conception bermanienne du traduire est đéjoué par un historicisme que le groupe a pleinement assumé : il lui était impossible de faire autrement, à moins de contrevenir aux fondements mêmes de son projet de traduction et d'effacer (ou ennoblir), à l'instar des pratiques traductives dominantes, les sociolectes faulknériens. Voilà, me semble-t-il, une première reconceptualisation de la traduction littérale bermanienne qui a permis au groupe de maintenir le caractère progressiste (non élitiste, non conservateur) de son projet de traduction.

Mais il en est une deuxième, qui a plus particulièrement trait à la politique de dévernacularisation. On l'a vu : a priori indice du rejet de soi au profit de l'autre, la survernacularisation pratiquée au début du projet véhiculait, malgré la politique de décentrement dont elle procédait, une image on ne peut plus familière de soi-du Québécois, de son parler, ses ambiguittés identitaires, ses revendications culturelles -, submergeant l'altérité du texte de Faulkner par des considérations par trop domestiques. Parallèlement, la survernacularisation a obligé le groupe de faire dévier le travail sur la lettre, le texte de Faulkner proposant un nombre nettement plus restreint de marqueurs vernaculaires. C'est dire que la survernacularisation correspondait bien à une forme d'exotisation (en rajouter pour faire plus vrai) que la politique de dévernacularisation n'a eu cesse de rectifier afin de respecter, précisément, les princípes de toute traduction littérale. Mais ce faisant, la survernacularisation a eu pour effet - bénéfique - d'attirer l'attention du groupe sur l'incontournabilité des questions de lisibilité et de lectorat, dans la mesure où l'on s'est rendu compte qu'une vernacularisation trop appuyée risquait d'inviter des réactions, fâcheuses dans une perspective bermanienne, de non-adhésion et de repli sur soi : malaise chez les lecteurs québécois et rire 
condescendant de la part des lecteurs non québécois qui refuseraient dès lors toute ouverture sur l'Autre.

Le processus de dévernacularisation reposait donc explicitement (mais non exclusivement) sur des problèmes de réception, avec pour conséquence que la retraduction accorde aux lecteurs une place et un rôle de fait, installant au centre d'une pratique traduisante littérale des figures de lecteur et des positions de lecture qui sont partie intégrante des stratégies traductionnelles du groupe. On est loin du rejet radical du lecteur prôné par Berman, et beaucoup plus proche de la perspective de Robinson dans The Translator's Turn, pour qui les traductions litterales ne sauraient pas ne pas agir sur le lecteur, de par le fait qu'elles sont

[...] motivated by serious ethical concerns - specifically, a concern to avert cultural facility, cultural superficiality, cultural aridity [...] : through an ostensible expression of aversion for the * reader $n$, the translator pragmatically teaches the real reader aversion for [...] a submissive role that encourages interpretation along institutionally approved lines. [...] Once this aversion [...] has been conveyed, then [... it] takes on enormous transformative power - the power to defamiliarize the familiar landscape of ideosomatic [institutionalized] world views, to articulate the buried inarticulated glimmerings of another reality (an unprogrammed experience $[\ldots]$ ), and to move the reader toward accessing that reality. [...] Translation as liberation. (Robinson, 1991, pp. 247-49)

L'étrangeté, même si elle a été diluée par le processus de dévernacularisation, des passages traduits en québécois assure, justement, ce rôle essentiellement transformateur, iconoclaste et libérateur par rapport aux habitudes, valeurs et catégories du savoir des lecteurs empiriques.

En accordant une place et un rôle de fait aux lecteurs, non seulement le groupe a rompu, a nouveau, avec la conception bermanienne de la traduction littérale, mais il a modifié en la déplaçant la notion, toute aussi bermanienne, de la visée éthique de la traduction. Et voici la troisième reconceptualisation importante effectuée par le groupe. Pour Berman, il existe une visée éthique négative (hypertextualité, domestication, ethnocentrisme) associée aux pratiques traductionnelles fidèles au sens du texte source, à laquelle s'oppose une visée éthique 
positive (neutralisation de l'ethnocentrisme, étrangeté, ouverture sur l'Autre) associée aux pratiques traductionnelles fidèles à la lettre du texte source. D'après lui, ces deux visées éthiques sont constitutives de l'acte de traduction qu'elles règlent pour ainsi dire de l'intérieur, grâce à un système axiologique dualiste exprimable en termes de bonne/mauvaise traduction : a J'appelle mauvaise traduction la traduction qui, généralement sous couvert de transmissibilité, opère une négation systématique de l'étrangeté de l'œuvre étrangère * (Berman, 1984, p. 17).

La politique de dévemacularisation du groupe démasque la relativité d'une telle dichotomie. D'une part, la survernacularisation a clairement montré que certaines * foreignizing strategies * fondées sur un travail sur la lettre (une politique de décentrement) sont susceptibles de verser, à l'insu du traducteur, dans l'ethnocentrisme et la domestication. D'autre part, le processus de dévemacularisation amorcé afin de corriger une telle entorse aux préceptes de la traduction littérale, tout en relevant d'une standardisation linguistique perçue comme ethnocentrique, et tout en prenant en considération les attentes du lecteur cible (tactiques de réappropriation), est paradoxalement moins ethnocentrique, plus fidèle aux systématismes - à l'étrangeté - du Hamlet.

Il s'ensuit que la ligne de démarcation entre visées éthiques négative et positive derneure foncièrement indéterminée, ce qui oblige à repenser à la fois la notion d'une éthique de la traduction et les conditions de possibilité mêmes de la $\alpha$ fidélité »a la lettre du texte original; quels que soient les efforts déployés pour en respecter l'étrangeté. Berman le dit lui-mềme : aucun vernaculaire d'arrivée ne saurait être équivalent (fidèle) au vernaculaire de départ, si bien que le traducteur ne peut qu'assumer les pertes et les gains massifs - linguistiques, idéologiques, politiques, rhétoriques - que déclenche invariablement la traduction des vernaculaires litterraires selon des modalités toujours plus ou moins acclimatisantes. Mais ce faisant, le traducteur se doit de prendre position par rapport à ces pertes et ces gains, dans un mouvement proprement éthique qui découle non pas de l'acte de traduire en soi mais bien du code éthique auquel le traducteur adhère en tant qu'agent historiquement déterminé, certes, mais néanmoins responsable de ses choix. C'est de ce code éthique que découlent son positionnement relatif aux vernaculaires source et cible, sa position traductive et les stratégies traductionnelles qui 
en émanent ${ }^{16}$. En définitive, le groupe n'a jamais cherché à refuser ni la responsabilité des pertes/suppléments de sens occasionnés par ses décisions traductionnelles, ni les incongruences idéologiques et historiques provoquées par le recours au parler rural québécois. Au contraire, les modifications peu à peu apportées aux fondements élitistes et idéalistes de la conception bermanienne de traduction littérale revêtaient une nécessité interne au projet pour autant qu'elles permettaient à la fois de ne pas en compromettre la portée contestataire et de réduire les contradictions idéologiques qui travaillaient le projet traductif de départ.

Entreprise protofasciste de coercition politique? J'en doute : entreprise libérale et démocratique, plutôt, fondée sur une volonté de démarginalisation, d'individuation et de différenciation. Pratique traductionnelle autoritaire et monologique? Je ne le crois pas, l'évolution des stratégies traductionnelles ayant permis au groupe de rester fidèle à son rôle d'instance traduisante désireuse d'entretenir un dialogue non seulement avec ses lecteurs, mais, à un plan plus spécifiquement méthodologique et conceptuel, avec les écrits de Berman, lesquels se trouvent pris dans ce mouvement dialogique d'ouverture et de métissage dès lors généralisé qui, selon moi, constitue la profonde originalité du projet" . Littéralisme timide, qui n'a pas le courage de ses convictions? Je ne le crois pas non plus, les stratégies traductionnelles du groupe l'ayant conduit à échapper aux dangers d'un littéralisme trop radical, constamment sur le point de reverser dans un ethnocentrisme d'autant plus insidieux que, refusant les voies conventionnelles de

I6 Dans son œuvre posthume, Pour une critique des traductions : John Donne (1995), Berman assouplit considérablement sa definition de ce qu'il nomme désormais l'éthicité (et non plus la visée éthique) de la traduction.

${ }^{17}$ A cet égard, il me semble que la retraduction du groupe actualise ce qui, selon Robinson, n'est que virtuel dans les écrits de Berman, à savoir :

[...] a potential openness [...] to all forms of transformative translation (except of course reductive, assimilative, ethnocentric ones) that would break down the barriers between the domestic and the foreign, the self and the other; words and ideas flow uneasily across those cuts, contaminating each other, undermining all pretensions of purity. (1997, p. 85) 
1'hypertextualité, il emprunte celles, nettement plus iconoclastes, du décentrement et de l'étrangeté. Entreprise aliénante et mystificatrice? Nullement, la retraduction proposée n'étant ni élitiste ni discriminatoire en ce sens qu'elle se fonde sur la mise en phase de positions de lecture non hiérarchisées que peuvent venir occuper aussi bien un lectorat spécialisé qu'un lectorat non spécialisé. Enfin, entreprise qui, sous le couvert d'une politique de décentrement, déploie avant tout des tactiques de réappropriation? Sans doute, à condition d'y voir ni une incohérence, ni une contradiction, mais un processus à la fois inhérent à l'acte traductionnel et pleinement assumé par le groupe.

Car il me semble que, en fait d'érrangeté, ce n'est qu'en donnant droit de cité à l'étranger du dedans qu'il est possible d'ouvrir une brêche à partir de laquelle une appréhension de l'étranger du dehors - aussi médiatisée soit-elle sur les plans culturel et institutionnel - peut être envisagée. Du coup, se trouvent respectées non seulement l'altérité des textes source et d'arrivée, mais aussi l'altérité du lecteur cible, lui-même étranger, à des degrés variables il est vrai, aux deux. Dans cette optique, la distinction éthique établie par Robinson entre le néolittéralisme (qu'il rejette) et la domestication (qu'il revendique), qu'ils soient timides ou radicaux, me paraît tout à fait discutable :

Radical literalism [...] creates an almost unreadable text that a highly trained bilingual reader reads like Finnegans Wake [...] one that, because it is unreadable to the uninitiated, is ineffective in the authoritarian project of stupefaction to which the neoliteralists unwittingly, or at least without admitting it, lend their support. Disturbing domestication of all sorts, from archaized and modernized to overtly propagandistic renditions, can be read, enjoyed, and raged at by everybody; as such it still remains the most effective way to unsettle the complacent reader. (Robinson, 1997, p. 96)

Outre le fait qu'un archaîsme (stratégie de domestication) peut être aussi illisible (ou " unsettling ") pour le lecteur non initié qu'une agrammaticalité (stratégie littérale), la dichotomie établie ici par Robinson, comme tant d'autres dichotomies qui postulent une * bonne * et une " mauvaise " manière de traduire, escamote ce qui me semble constituer la complexité, ou encore l'hybridité de l'acte traductionnel, sans cesse aux prises avec le propre et l'autre, soi et l'étranger, la possibilité du littéralisme et l'inévitabilité de la domestication. En dernière 
analyse, il me paraît futile de vouloir, à tout prix, tracer des lignes de démarcation trop précises. Comme j'ai tenté de le démontrer dans les pages qui précédent, le choix de recourir au vernaculaire rural québécois pour respecter ce que Berman nomme les systématismes du texte source, son étrangeté, engage à la fois, selon un rapport d'implication mutuelle, une stratégie littérale de décentrement socio-culturel (au sens de Berman) et des tactiques de domestication (au sens de Robinson) dont la finalité commune consiste à refuser l'hypertextualité et l'ethnocentrisme radical en bafouant les attentes du lecteur - que son état soit de stupeur ou de complaisance. Tout au plus, seul sera susceptible de varier, au sein des passages en vernaculaire québécois, le dosage du littéral, avec son coefficient plus ou moins élevé d'illisibilité, et de la domestication, avec son coefficient plus ou moins élevé d'anachronismes et d'incongruités.

Université McGill

\section{Références}

BARTHES, Roland (1964). "Qu'est ce que la critique? ", in Essais critiques, Paris, Seuil, pp. 51-68.

BERMAN, Antoine (1984). L'épreuve de l'étranger, Paris, Gallimard.

BERMAN, Antoine (1985a). * La traduction comme épreuve de l'étranger $n$, in Texte 4, pp. 67-81.

BERMAN, Antoine (1985). « La traduction et la lettre - ou l'auberge du lointain n, in Antoine Berman et al. (éds), les tours de Babel, Mauvezin, Éditions Trans-Euro-Repress, pp. 31-150.

BERMAN, Antoine (1995). Pour une critique des traductions : John Donne, Paris, Gallimard.

BOUCHARD, Chantal (1988). * De la "langue du grand siecle" à la "langue humiliée", les Canadiens français et la langue populaire, 1879-1970 , in Recherches sociographiques, vol. 29, $\mathrm{n}^{\circ} 1$, pp. 7-21. 
BOUCHARD, Chantal (1990). « Contes et légendes du Canada français : le mythe du French Canadian Patois ", in Bulletin de l'Assaciation canadienne de linguistique appliquée, vol. $12, \mathrm{n}^{\circ} 1$, pp. 35-49.

BOUCHARD, Chantal (1998). La langue et le nombril. Histoire d'une obsession québécoise, Montréal, Fides.

BRISSET, Annie (1990). Pour une sociocritique de la traduction. Théatre et altérité au Québec (1968-1988), Longueuil, Québec, Les Éditions du Préambule.

CHAPDELAINE, Annick et Gillian LANE-MERCIER (dirs), avec la collaboration de Corinne Durin, Christiane Mayer, Bernard Vidalt, Lucie Joubert, Peter Di Maso, Sophie Boivin, Judith Lavoie, Hélène Buzelin, Michael Gilson. Retraduire The Hamlet de Foulkner ; la réflexion à l'auvre, la réflexion par l'auvre, (à paraître).

LANE-MERCIER, Gillian (1995). * La traduction des discours directs romanesques comme stratégie d'orientation des effets de lecture $»$, in Palimpsestes 9, pp. 75-91.

LANE-MERCIER, Gillian (1995). * Toward a Rhetorical Practice of Mimesis : Writing/Reading/(Re)translating Fictional Sociolects. ", in Recherches sémiotiques/Semiotic Inquiry, vol. 15, $\mathrm{n}^{\circ} 3$, pp. 105-128.

LANE-MERCIER, Gillian (1997). * Translating the Untranslatable : The Transtator's Aesthetic, Ideological and Political Responsibility ", in Target vol. 9, no 1, pp. 43-68.

MASSARDIER-KENNEY, Françoise (1994). * Translation Theory and Practice ", in Doris Kadish and Françoise Massardier-Kenney (eds), Translating Slavery, Gender and Race in French Women's Writing, 1793-1823, Kent, OH, Kent State University Press, pp. 11-25.

ROBINSON, Douglas, (1991). The Translator's Turn, Baltimore and London, The Johns Hopkins University Press. 
ROBNSON, Douglas (1997). What is Translation? Centrifugal Theories, Critical Interventions, Kent, Ohio, and London, England, The Kent State University Press.

SIMON, Sherry (1992). "The Language of Cultural Difference : Figures of Alterity in Canadian Translation $"$, in Lawrence Venuti (ed.), Rethinking Translation. Discourse, Subjectivity, Ideology, London and New York, Routledge, pp. 159-176.

VENUTI, Lawrence (ed.) (1992). Rethinking Translation. Discourse, Subjectivity, Ideology, London and New York, Routledge.

VENUTI, Lawrence (1995). The Translator's Invisibility. A History of Translation, London and New York, Routledge.

RÉSUMÉ : Le travail sur la lettre : polítique de décentrement ou tactique de réappropriation ? - La visée de cet article est double. D'une part, il s'agit de répondre à quelques-unes des attaques récentes dirigées par Douglas Robinson contre les pratiques traductionnelles littérales, que Robinson qualifie de « protofascistes ». D'autre part, il s'agit d'interroger, dans le cadre d'un projet de retraduction littérale de The Hamlet de William Faulkner mené à l'université MoGill entre 1990 et 1996, la valeur épistémologique, éthique et méthodologique de l'approche littérale prônée par Antoine Berman, en vue à la fois d'une remise en question et d'une reconceptualisation de cette demière, dont l'élitisme et l'idéalisme ont déjà été soulignés. Pour ce faire, nous proposons une analyse des présupposés politico-idéologiques véhiculés, d'un côté, par le recours au vernaculaire rural du Québec pour traduire le parler non-standard des personnages faulkneriens, et de l'autre, par les stratégies de survernacularisation et de dévernacularisation successivement mobilisées a ces fins. $\mathrm{Si}$, à première vue, ces stratégies correspondent, respectivement, à une politique de décentrement (littéralisme, nonannexion de l'Autre socio-culturel) et à une tactique de réappropriation (hypertextualité, domestication de l'Autre socio-culturel), l'analyse démontre jusqu'à quel point une telle distinction est peu opératoire sur les plans épistémologique, ethique et heuristique. En effet, le choix de recourir au vernaculaire rural québécois pour respecter l'étrangeté du texte source engage simultanément, selon un rapport d'implication mutuelle, le 
littéralisme et la domestication, dont la finalité commune consiste à refuser l'ethnocentrisme radical en déstabilisant les attentes du lecteur.

\begin{abstract}
The Politics and Tactics of Literal Translations : Foreignism or Reappropriation ? - The objectives of this article are twofold. On the one hand, it seeks to respond to some of the attacks recently leveled by Douglas Robinson at literal translational practices, which he defines as " protofascist $»$. On the other hand, referring to a project conducted at McGill University between 1990 and 1996 in which a literal retranslation of William's Faulkner The Hamlet was undertaken, it at once questions the epistemological, ethical and methodological value of Antoine Berman's conception of literalism, and shows how the latter can be reconceptualized in order to avoid the elitism and the idealism for which Berman has, on occasion, been criticized. In this perspective, an analysis of the political and ideological assumptions underlying not only the choice of Québec rural vernacular to translate the non-standard speech of Faulkner's characters, but also the strategies of over-vernacularisation and de-vernacularisation successively mobilized for such a purpose, is proposed. If, in the first instance, these stategies appear to entail, respectively, foreignism (i.e. literalism, the non-colonization of the sociocultural Other) and reappropriation (i.e. fluency, the domestication of the socio-cultural Other), the analysis shows to what extent such a distinction is of little epistemological, ethical and heuristic value. Indeed, the choice to respect the foreignness of the source text by using Québec rural vemacular entails a reciprocal relation of literalism and domestication, the common goal of which is to reject radical ethnocentrism via the destabilization of reader expectations.
\end{abstract}

Gillian Lane-Mercier : Département de langue et littérature françaises, Université McGill, 3460, rue McTavish, Montréal (Québec) H3A 1 X9. 